D. Ayres

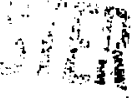


THE EFFECTIVE MASS SPECTROMETER

\author{
D. Ayres \\ Argonne National Laboratory, Argonne, IL 60439
}

\title{
ABSTRACT
}

The history and major accomplishments of the Effective Mass Spectrometer (EMS) are described. In the eight years since the EMS turned on, 21 experiments have been completed by groups from nine institutions in 32 months of operation. Over 400 million triggers have been recorded on magnetic tape, resulting in 29 journal publications to date. A list of experimental proposals for the EMS and a sampling of results are presented.

\section{THE EARLY DAYS}

As I was struggling to gain some sort of historical perspective on the Effective Mass Spectrometer for this talk, it occurred to me that I would be speaking to you on a personal anniversary of some significance. Just ten years ago tomorrow I was arriving at Argonne to begin work with the new Spectrometer Group, which then consisted of Bob Diebold, Art Greene, Barry Wicklund and myself. Looking back through my notes of that time, I found that the Effective Mass Spectrometer was no more than a collection of back-of-the-envelope scribblings, Fermilab was only a muddy hole in the ground, Meson Spectroscopy was in its heyday, the $\mathrm{A}_{2}$ was split, and gasoline was 35 cents a gallon!

Meson spectroscopy, and the split $A_{2}$ in particular, had a profound effect on the design of the EMS, and determined many of the properties which have been responsible for the success of the spectrometer. Thus, one of our design goals was to be able to study the $\mathrm{K}^{*}$ (1420), in case other tensor mesons might also be split. To this end we labored to obtain simultaneously good effective-mass resolution and large acceptance at high masses, and to design a short beamline with lots of $\mathrm{K}$ mesons, good particle identification, and good momentum resolution. Although the $\mathrm{K}^{*}(1420)$ experiment had become uninteresting by the time we were able to perform it, the stringent requirements which it imposed on the design have been very important for the versatility and productivity of the spectrometer facility. In return, our debt to meson spectroscopy was amply repaid by $\mathrm{E}-268$, a comprehensive comparison of $\mathrm{K}^{*}$ and $\rho$ production, and a precise measurement of $p-w$ interference.

Early on in our design studies we began to collaborate closely with members of Drasko Jovanovic's Wire Spark Chamber Group, which also included Dave Rust and Charlie Ward. Their experience with online computer data acquisition systems, spark chamber construction and operation, and with the previous generation of spectrometers at the ZGS, provided much of the technical foundation upon which the EMS was built. As I mention a few of the names of those who played major roles in the early

Talk presented at the Symposium on the History of the ZGS, Sept. 13-14, 1979, at Argonne National Laboratory. 
days of the EMS, I realize that I am leaving a lot of people out. In an attempt to rectify this, I have tried to list in $\mathrm{Table} I$ many of those who contributed to the EMS at one time or another.

Table I: Major Contributors to EMS Construction

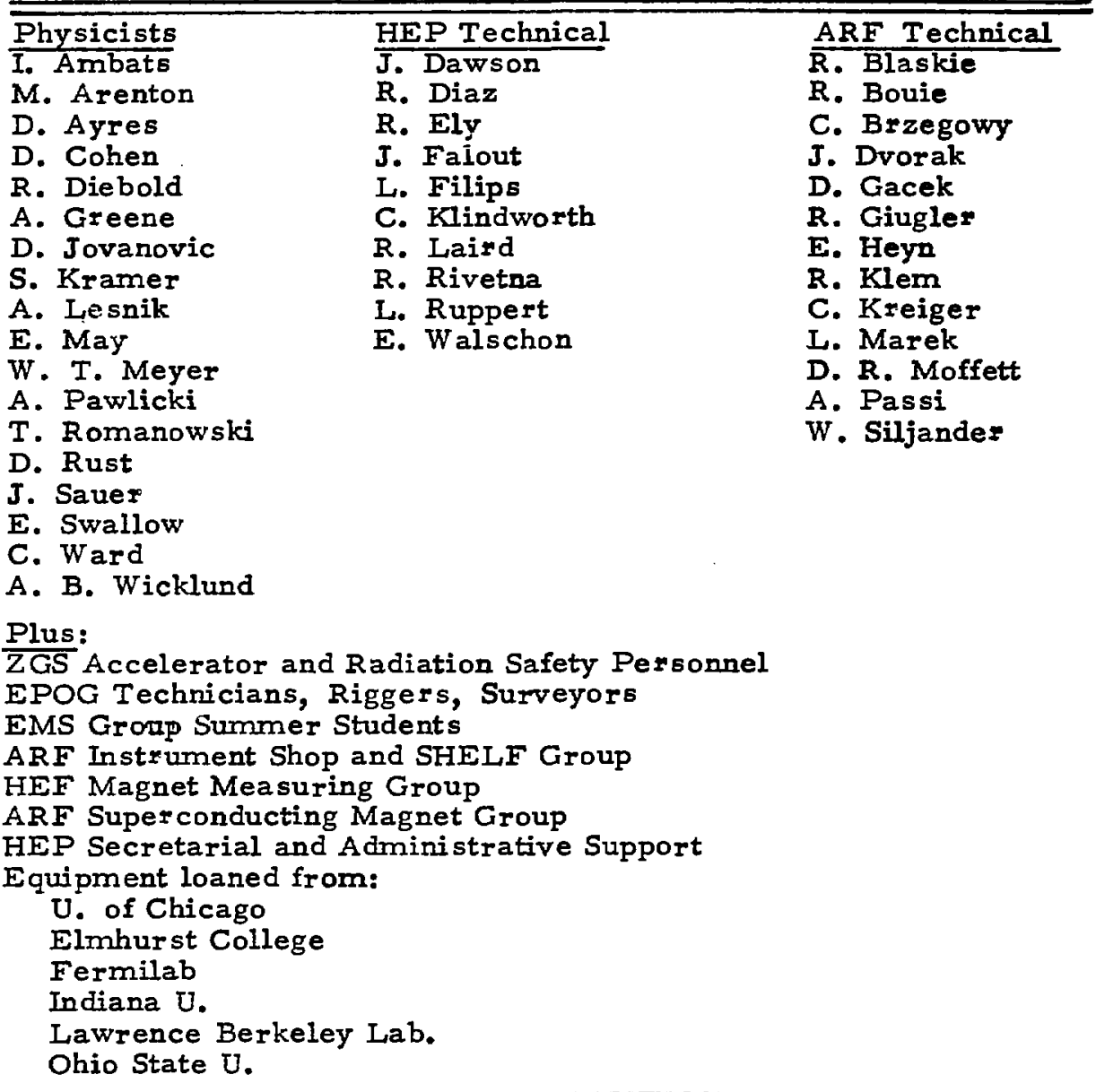

Figure 1 shows the assembled members of the two groups which built the EMS, shortly after the start of operations in the summer of 1971 . The photograph also reveals (in the background) another reas on why the EMS has been so versatile - each group had its own hydrogen target cart as sembly mounted on a set of rails, so that the targets and surrounding detectors could be ea sily interchanged. The versatility of the Spectrometer as a facility in future years was in large part due to the fact that it was originally designed to accommodate two rather different experiments: the Diebold Group's vector-meson production studies, and the Jovanovic Group's investigations of associated production. 


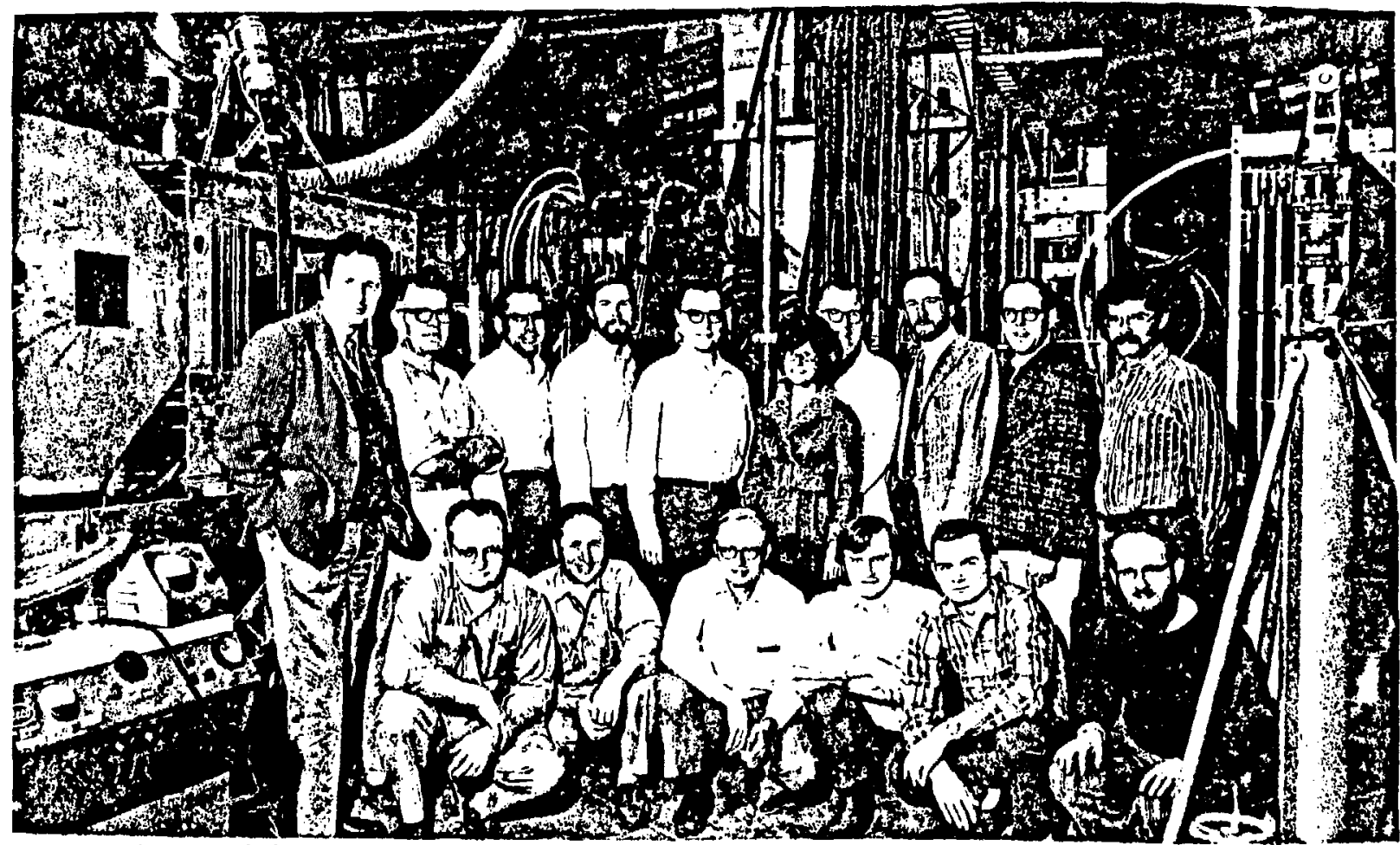

Fig. 1. Builders of the original EMS. From left to right: (rear row) D. Jovanovic, E. Walschon, D. Ayres, W. T. Meyer, C. Ward, R. Rivetna, L. Ruppert, A. B. Wicklund, A. Greene, A. Lesnik; (front row) L. Filips, R. Diaz, R. Diebold, I. Ambats, S. Kramer, D. Rust. The spectrometer magnet can be seen in the center background, the Jovanovic-Group hydrogen target cart is in place in the beamline (to the left of the magnet), and the Diebold-Group target cart is pushed out of the beamline (far left). The beam direction is from left to right. 


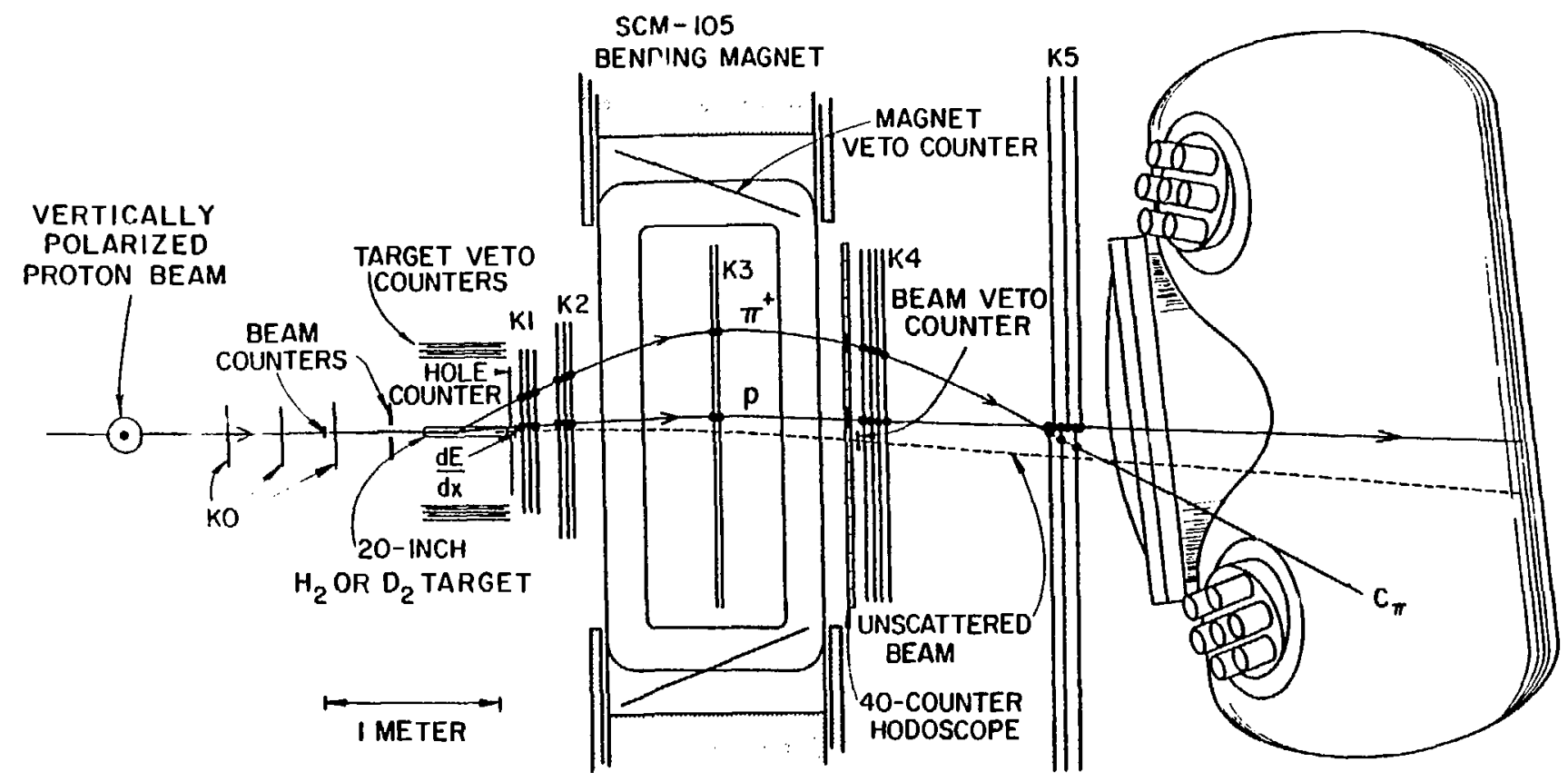

Fig. 2: Plan view of the EMS as it was used in E-339 for a study of $\mathrm{p}_{\mathrm{p} \rightarrow \Delta}{ }^{++} \mathrm{r}$. with the polarized proton beam. K0, K1, K2, K3, K4, K5 are sets of magnetostrictive-readout spark chambers. The large downstream Cerenkov $C_{\pi}$ distinguished between $p$ and $\pi^{+}$. 


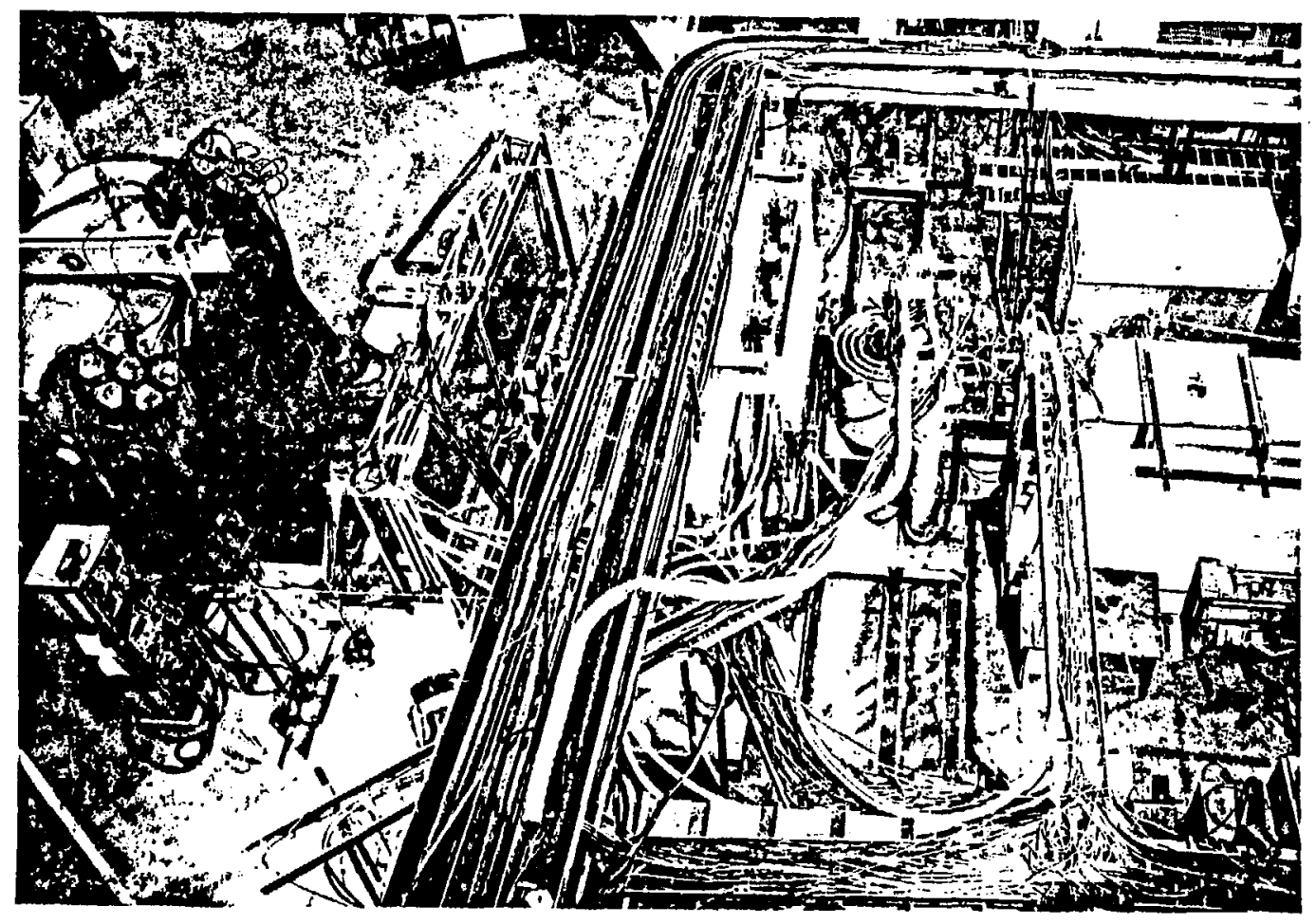

Fig. 3. A photograph of the EMS as it was used for $E-346$, to study $\pi^{-} p \rightarrow K^{0} \Lambda$. The Jovanovic-Group
target cart, with the cylindrical gpark chambers target cart, with the cylindrical spark chambers, is installed in the beamline, and the Diebold-Group target cart, with itg array of target veto counters, is rolled out to the right of the beam. The beam 


\section{EVOLUTION OF THE EMS}

In order to describe the evolution of the EMS, I will first need to review briefly some of the basic features of its construction. Figure 2 shows a plan view of the EMS, essentially a large aperture dipole magnet (11 $\mathrm{kG}-\mathrm{m}$ ) surrounded by magnetostrictivereadout spark chambers. The basic elements of the trigger were the 40 -counter hodoscope which counted particles emerging from the magnet, a beam veto counter, and veto counters surrounding the magnet aperture and hydrogen target. Experiments studying $K^{\circ}$ and $\Lambda$ production used pulse height counters to define both ends of a decay space downstream of the target. The large downstream Cerenkov counter in Fig. 2 separated final-state pions from protons. It was borrowed from the University of Chicago for E-325, a study of $\mathrm{K}^{+} \mathrm{K}^{-}$production, to veto events with final-state pions. Figure 3 is a photograph of the EMS showing the cylindrical spark chambers which were used in E-331 and E-346 to detect recoil protons, $A$ 's, and $\Delta^{\prime} s$. In the most recent configuration of the spectrometer, we have constructed a "Vertex Detector" from cylind,ical proportional-wire chambers to detect recoil particles and to permit triggering on charged recoil multiplicity.

Over the years the original EMS detectors were gradually upgraded or replaced, and its versatility and reliability steadily improved. Although the $K 1$ and $K 2$ chamber sets have withstood the entire 8 years of operation, all other chamber sets were replaced at one time or another: Table II outlines a few of the major milestones in the EMS evolution. Table III summarizes a few numbers

Table II: Milestones in the Evolution of the EMS

\begin{aligned} & $1969-1971:$ Design and Construction \\ & 1972: Large Downstream Cerenkov Counter Installed \\ & 1973: Cylindrical Spark Chambers Operational \\ & 1973: First Polarized Beam Experiments \\ & 1975: 12 GeV/c Superconducting Beamline Cornmissioned \\ & 1978: Vertex Detector and PDP-1134 Installed \\ & 1979: $\begin{array}{l}\text { Spin Rotating Solenoids Provide Longitudinal Beam } \\ \text { Polarization }\end{array} \\ &$\hline\end{aligned}

Table III: EMS Experiment Statistics

\begin{tabular}{lc}
\hline \hline Years of Operation (May, 1971 - August, 1979): 8.3 years \\
Number of Submitted Proposals: & 23 proposals \\
Number of Completed Experiments: & 21 experiments \\
Number of Institutions on Proposals: & 9 institutions \\
Number of Physicists on Proposals: & 56 physicists \\
Total Number of Triggers on Tape: & $4 \times 10^{8}$ triggers \\
Total Number of Shifts Charged: & $1925=32$ months \\
Published Journal Articles to Date: & 29 publications \\
\hline \hline
\end{tabular}




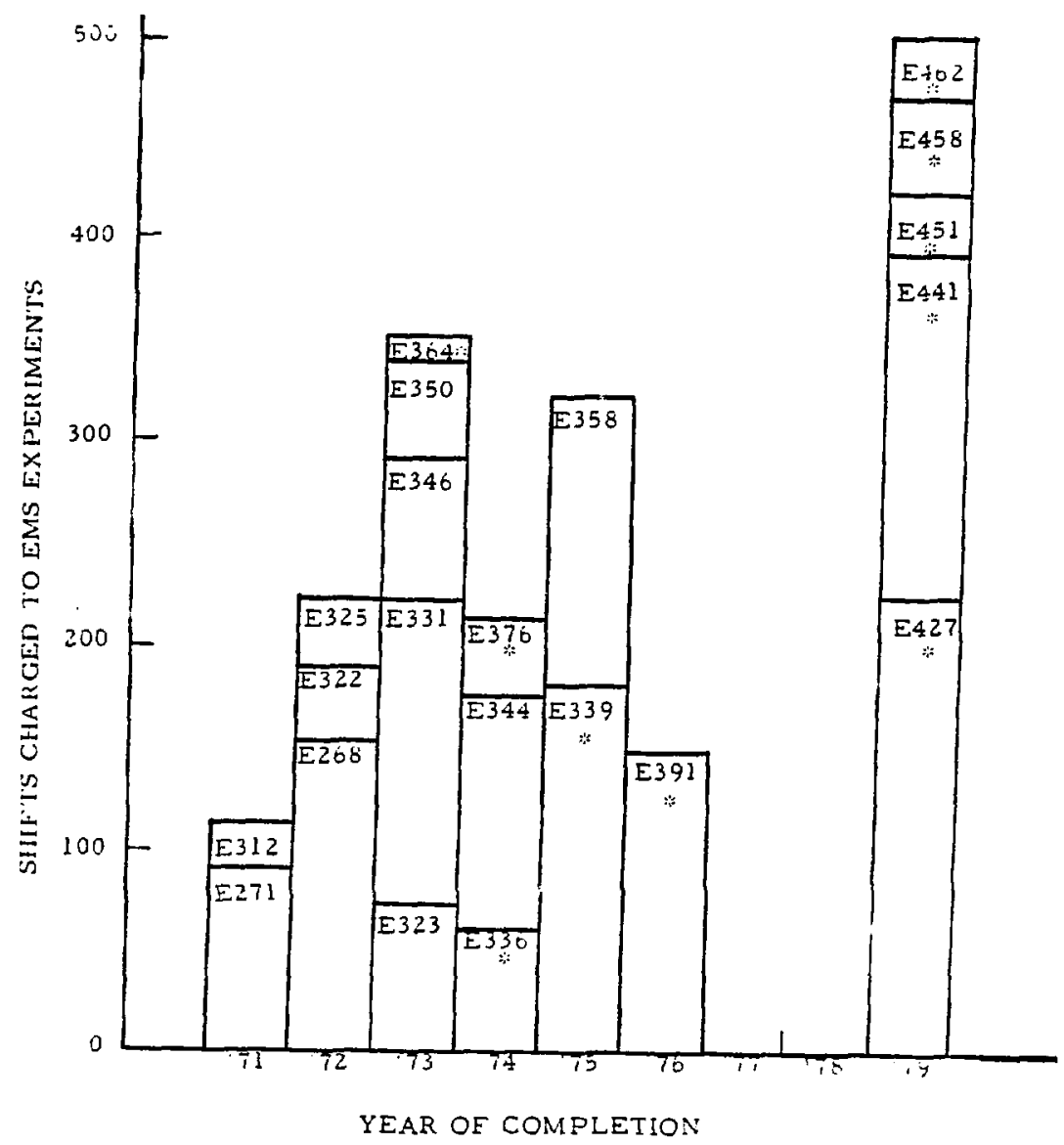

Fig. 4: Time distribution of EMS experiments. All shifts for each experiment are shown in the year when the experiment was completed. Asterisks indicate polarized beam experiments. 
which are some measure of the spectrometer's achievements in the past eight years. Of course the number of publications from the EMS will increase considerably as we dig into the large volume of new polarized beam data recorded during the last eight months of operation in 1979. Figure 4 gives an idea of the time distribution of EMS experiments, which are listed in detail in Table IV. During the gap in operations in 1977 and 1978, the beamline to the EMS was rebuilt for the third time, to allow the installation of spin-rotating solenoids for longitudinal beam polarization, and the Vertex Detector was built and installed.

Table IV: List of EMS Experiments

E-268 Proposal to Study Meson Production with a Wire-Spark-

Chamber Spectrometer (ANL), R. Diebold

Proposal - Jan. 1970, Completed - Jan. 1972, 155 shifts

E-27i A Proposal to Study the Reactions $\pi^{-} \mathrm{p}+\mathrm{K}^{0} \Lambda^{0}$ and $\pi^{-} \mathrm{p}+\mathrm{K}^{0} \Sigma^{0}$ in the Range 3.0 to $6.0 \mathrm{GeV} / \mathrm{c}$ (ANL), C. E. W. Ward Proposal-Feb. 1970, Completed-Dec. 1971, 89 shifts

E-312 Proposal to Measure Elastic Scattering at Small Angles (ANL), D. R. Rust

Proposal - Sept. 1.971, Completed - Oct. 1971, 23 shifts

E-322 Study of $\Lambda^{\circ}$ Production in $6 \mathrm{GeV} / \mathrm{c}$ Proton-Proton Interactions (U. of Chicago, ANL, Ohio State U.) T. M. Knasel Proposal - Jan. 1972, Completed - Feb. 1972, 35 shifts

E-323 A Measurement of the Three Pion Mass Spectrum in the Reaction $\pi^{-} \mathrm{C}^{12}+\pi^{+} \pi^{-} \pi^{-} \mathrm{C}^{12^{*}}$ (4.45 MeV) (U. of Mlinois), U. E. Kruse Proposal - Feb. 1972, Completed - Jan. 1973, 73 shifts E-325 Proposal to Study $f^{\circ}-A_{2}^{0}$ Interference in $\pi^{-} P^{+}+K^{+} K^{-} n$ (ANL), D. S. Ayres Proposal-March 1972, Completed - April 1972, 31 shifts

E-331 Proposal to Study the Reactions $K^{+} p+K^{\circ} \Delta^{++}$and $K_{n}^{-} \rightarrow K^{-} \Delta^{-}$ Using the Effective Mass Spectrometer (ANL, Calif. Inst. Tech.), B. Musgrave Proposal - March 1972, Completed-Dec. 1973, 148 shifts

P-332 Study of Multiple Pion Productions, Associated with Nuclear Gamma Transitions, (USCD), O. Piccioni Proposal - March 1972, (withdrawn) 
Table IV (continued): List of EMS Experiments

E-336 Measurement of the Polarization of $\Lambda^{\prime} s$ Produced by a Polarized Proton Beam (U. of Chicago, Ohio State U., ANI), R. Winston

Proposal - May 1972, Completed - May 1974, 59 shifts

E-339 Study of Resonance Production with a Polarized Proton Beam Using the Effective Mass Spectrometer (ANL), A. B. Wicklund

Proposal - May 1972, Completed-April 1975, 180 shifts

E-344 Neutral Meson Spectrum Near $1000 \mathrm{MeV}$ (Iowa State U.), E. C. Peterson

Froposal - July 1972, Completed - April 1974, 116 shifts

E-346 A Proposal to Measure $\Lambda^{\circ}$ Polarization with High Statistics in $\pi^{-} \mathrm{P}+\mathrm{K}^{0} \Lambda^{0}$ at $5 \mathrm{GeV} / \mathrm{c}$ (ANL), C. E. W. Ward Proposal - July 1972, Completed - Dec. 1973, 71 shifts

E-350 Further Study of Forward Elastic Scattering (ANL), R. Diebold Proposal - Oct. 1972, Completed - Feb. 1973, 47 shifts

E-358 Study of the $K \bar{K}$ System Using the Effective Mass Spectrometer and a Deuterium Target (ANL), A. B. Wicklund Proposal - April 1973, Completed - May 1975, 140 shifts

E-364 Proposal to Measure the pp Elastic Scattering Polarization Parameter Using the Effective Mass Spectrometer and the Polarized Beam (ANL, Indiana U., Ohio State U., U. of Chicago), D. R. Rust

Proposal - July 1973, Completed - Oct. 1973, 6 shifts

E-376 Measurement of the Polarization Parameter for pn Elastic Scattering from 2 to $6 \mathrm{GeV} / \mathrm{c}$ (ANL), R. Diebold Froposal - May 1974, Completed - Oct. 1974, 35 shifts

E-391 Measurement of Polarization Effects Using the Effective Mass Spectrometer and Polarized Proton Beam at 9 and $12 \mathrm{GeV} / \mathrm{c}$ (ANL), S. L. Kramer Proposal - March 1975, Completed-Dec. 1976, 143 shifts 
Table IV (continued): List of EMS Experiments

P-394 Measurement of Spin-Rotation Parameters in $\Lambda^{\circ}$ Production at $12 \mathrm{GeV} / \mathrm{c}$ Using the Polarized Proton Beam and the Effective Mass Spectrometer (Ohio State U., U. of Chicago, ANL),

A. Lesnik

Proposal - June 1975, (withdrawn)

E-427 Proposal to Study Exclusive $\Lambda$-Production Reactions with the ZGS Polarized Proton Beam (ANI, Elmhurst College, Ohio State U.), D. Ayres

Proposal-Jan. 1977, Completed - July 1979, 205 shifts

E-441 Proposal to Study the Reaction $\mathrm{P}_{\mathrm{P}} \mathrm{P} \rightarrow \mathrm{p} \pi^{+} \pi \pi^{-} \mathrm{P}$ with the $\mathrm{ZGS}$ Polarized Proton Beam (ANL), A. B. Wicklund Proposal - Oct. 1977, Completed-April 1979, 181 shifts

E-451 Proposal to Study Longitudinal Spin Correlations in the Reaction $\mathrm{pp} \rightarrow \mathrm{p} \pi^{+} \mathrm{n}$ at 6 and $12 \mathrm{GeV} / \mathrm{c}$ (ANL), A. B. Wicklund Proposal - Sept. 1978, Completed - June 1979, 41 shifts

E-458 Proposal to Study $\mathrm{pK}^{+} \mathrm{K}^{-} \mathrm{p}$ and $\mathrm{pPpp}$ Final States Using the Effective Mass Spectrometer (ANL, Elmhurst College),

M. Arenton

Proposal - Feb. 1979, Completed - May 1979, 48 shifts

E-462 Proposal to Study pp $\rightarrow \mathrm{pn}^{+}$Between 1.5 and $2 \mathrm{GeV} / \mathrm{c}$ (ANL, Elmhurst College, Rice U.) A. B. Wicklund . Proposal - July 1979, Completed - August 1979, 24 shifts

\section{AN EMS PHYSICS SAMPLER}

I could not possibly give a talk on the EMS without showing some data. Since time is short, I have simply chosen eight of my favorite figures to give you a sampling of the physics which has been produced so far. These are shown as Figs. 5 through 12. Because we've concentrated on recording as much data as possible before the ZGS slutdown next month, polarized beam results are somewhat under-represented in this collection. Within the next few years, however, these recent EMS experiments can be expected to add significantly to our knowledge: E-451 extended the study of $L^{++}$production at 6 and $12 \mathrm{GeV} / \mathrm{c}$ to include measurements with a longitudinally polarized beam, to help separate 
natural and unnatural parity-exchange production; E-46 2 continued these studies down into the threshold region with the help of the Vertex Detector, and holds the additional interest of perhaps detecting dibaryon resonances; $E-427$ was a high statistics study of $\mathrm{pN} \rightarrow \Lambda \mathrm{K}+\mathrm{N}$, where amplitude analyses and baryon spectroscopy will be constrained by the $\Lambda$ spin information, and where the use of proton and neutron targets isolates is ospin dependence; $E-441$ is expected to yield detailed information on baryon spectroscopy in $\mathrm{pp} \rightarrow \mathrm{p}^{+} \pi^{-} \mathrm{p}$ at 6 and $12 \mathrm{GeV} / \mathrm{c}$, with longitudinal beam polarization providing discrimination between production-mechanism asymmetries and resonance-decay asymmetries; $E-458$ was a measurement of $\mathrm{pp} \Rightarrow \mathrm{pK}^{+} \mathrm{K}^{-} \mathrm{p}$, $\mathrm{pp} \overline{\mathrm{p}} \mathrm{p}$, providing information on production mechanisms and baryon spectroscopy at high masses, and yielding a unique comparison of $\phi$ production and $\omega$ production (from pp $\rightarrow$ $\mathrm{p}^{+} \pi^{-} \pi^{\circ} \mathrm{p}$ in $\left.E-441\right)$ in $\mathrm{pp}$ interactions. Thus, although the Effective Mass Spectrometer is now being dismantled, you can expect EMS physics results to continue to emerge for several years to come.

\section{THE EMS IN PERSPECTIVE}

Now that the ZCiS program is drawing to a close I cannot help but ask what lessons there are to be learned from the amazing productivity of our simple spectrometer. Although the EMS had it's share of startup "debugging" and routine equipment failures, the actual accelerator time lost to such exercises was quite low due to the high level of technical support in the ZGS complex, and to the conservative design of the spectrometer itself. The relatively high reliability of the detectors was in large measure due to the use of well-proven technology - scintillation counters, Cerenkov counters, and spark chambers were already "old hat" when the EMS was being designed. A second essential element was that, despite numerous improvements and replacements, the basic EMS changed remarkably little over the years. Targets and detectors upstream and downstream of the magnet-spark-chamber system were changed, but the basic apparatus for measuring fast forward charged particles was always the same.

Throughout the first few years of EMS operation I found myself worrying that the big, multipurpose spectrometers at other accelerators would put us out of business as soon as they came into operation. The LASS spectrometer at SLAC, the CERN Omega, and the Brookhaven MPS were all more expensive and sophisticated devices than the EMS, but despite my concern, the EMS never did come into direct competition with them. Due to their size and complexity, these facilities took much longer to build, and thus began operation several years after the EMS. When they finally were ready to do physics, they often chose to take advantage of the higher energy beams available to them, and to study the more complex reactions which were accessible with their large acceptance and sophisticated triggering and tracking instrumentation. Their more ambitious goals seemed to further delay the output of physics results. Life at the ZGS turned out to be much simpler: reactions with only a few final-state particles were easy to detect 
and record, and had high cross sections at $Z G S$ energies. Triggers were easy because the reactions of interest were a relatively large fraction of the total cross section, and reconstruction of two - or three-track events was immensely simpler than high multiplicity final states. Because the scintillation counters, wire spark chambers, and Cerenkov counters of the EMS were not at the frontier of technology, they turned on quickly and performed reliably year after year. The device was so simple to operate, that university groups were able to perform experiments with only a minimum of instruction by Argonne physicists.

The choice of experiments also had a lot to do with our success: high event rates made it easy to perform high statistics, systematic studies of related reactions over the whole range of $Z$ GS energies. We utilized beams of $\pi^{ \pm}, K^{ \pm}$, protons and antiprotons, on both hydrogen and deuterium targets to isolate isospin dependence. Such comprehensive studies of related reactions with a single apparatus allowed us to perform comparisons with much smaller systematic errors than had been achieved before. It turned out that there were things to learn about even the simplest reactions. An excellent example of this is the first EMS experiment to be completed $(E-312)$, a measurement of elastic scattering of $\pi^{ \pm}, K^{ \pm}, p$ and $\bar{p}$ from protons, which measured the crossover values and their energy dependence very precisely. High statistics studies of meson spectroscopy and production mechanisms dominated the program in the early years, and this evolved naturally into a study of inelastic reactions using the polarized proton beam when it became available. With the polarized beam we were once again able to learn new things about simple reactions, a good example being the EMS measurement of the polarization parameter in proton-neutron elastic scattering (E-376).

The timely availability of the polarized proton beam was an important factor in the EMS productivity. As Fig. 4 illustrates, the polarized beam experiments joined smoothly onto the conclusion of the unpolarized beam program - had the polarized beam come a few years later, there might have been no EMS to make use of it. As it turned out, 10 of the 21 completed experiments utilized polarized protons.

\section{ACKNOWLEDGEMENTS}

I cannot end this talk without acknowledging the enormous contritution made by the ZGS staff, experimental area technicians, and support groups in ARF, $A D, H E F$, and HEP Divisions. Table I represents only the tip of a very large iceberg, and gives little hint of the long hours of hard work which were devoted to keeping the EMS on the air, usually by people who had to take the physicists ${ }^{\prime}$ word $f(r$ it that what we were doing made sense. It was the dedication of the people who worked here that ultimately made our work a success. If I may borrow the words of Bruce Cork, we were most fortunate to be working "at the right machine, at the right place, at the right time." 


\section{REFERENCES}

1. D. S. Ayres, in Proceedings of the Inte rnational Conference on Instrumentation for High Energy Physics, Frascati, Italy, 1973, edited by S. Stipcich (Laboratori Nazionali del Comitato Nazionale per l'Fnergia Nucleare, Frascati, Italy, 1973), p. 665.

2. I. Ambats et al., Phys. Rev. D9, 1179 (1974).

3. A. B. Wicklund et al., Phys. Rev. D17, 1197 (1978).

4. C. E. W. Ward etal., Phys. Rev. Lett. 31,1149 (1973).

5. J. J. Phelan et al., Phys. Lett. 61 B, 483 (1976).

6. D. S. Ayres et al., Phys. Rev. Lett. 32, 1463 (1974).

7. A. J. Pawlicki et al., Phys. Rev. Dl5, 3196 (1977).

8. R. Diebold et al., Phys. Rev. Lett. 35, 632 (1975).

9. S. L. Kramer et al., Phys. Rev. D17, 1709 (1978).

10. R. Diebold et al., in Proceedings of Orbis Scientiae 1977 on Deeper Pathways in High-Energy Physics, Coral Gables, Florida, 1977, edited by A. Perlmutter and L. F. Scott (Plenum Press, New York, 1977), p. 109.

\section{SUPPORT}

This work was performed under the auspices of:

The U. S. Atomic Energy Commission,

The U. S. Energy Research and Development Administration,

The U. S. Department of Energy. 


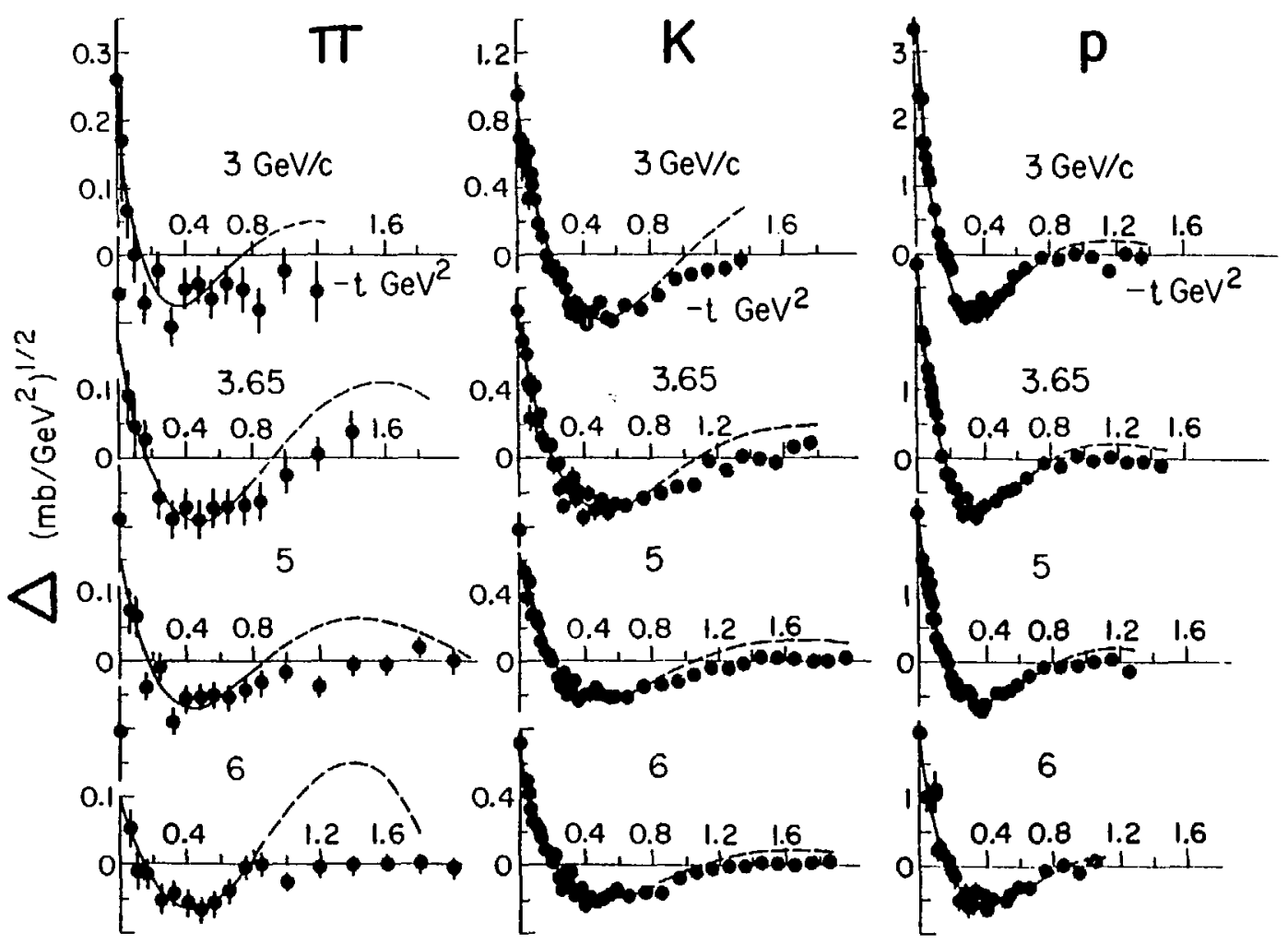

Fig. 5: Normalized elastic scattering cross-8ection differences $\Delta^{=}\left(\sigma^{-}-\sigma^{+}\right) /\left[8\left(\sigma^{-}+\sigma^{+}\right)\right]^{1 / 2}$. Curves are fits to a Besel function parametrization in the interval $0 \leq-t \leq 0.8 \mathrm{GeV}^{2}$. (From E-312: see ref. 2.) 


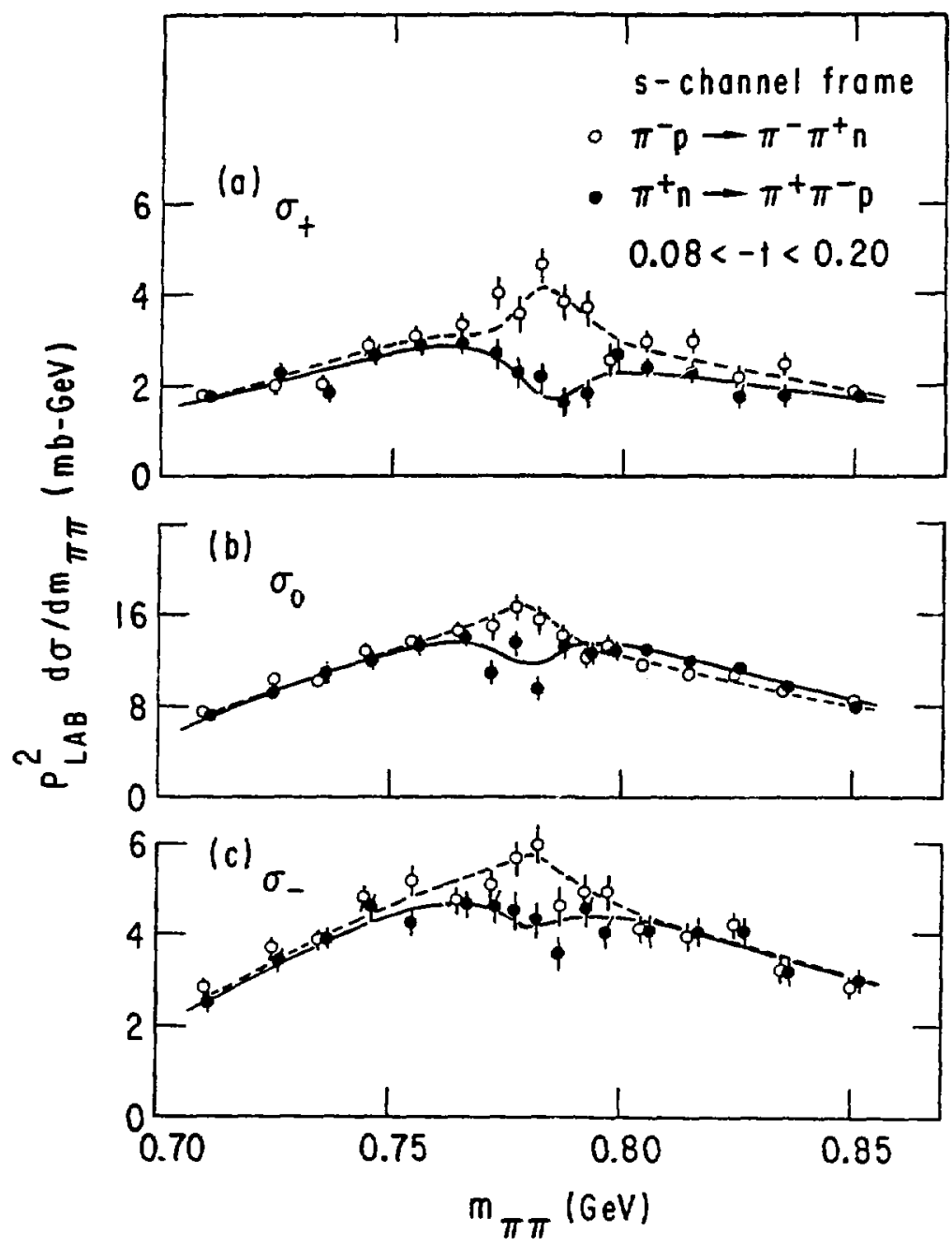

Fig. 6: Comparison of the dipion mass spectra in the $p$-w interference region for the reactions $\pi^{-} p+\pi^{-} \pi^{+} n$ and $\pi^{+} n \rightarrow \pi^{+} \pi^{-} p$, where the s-channel ${ }^{2}$ projections $\sigma_{0}, \sigma_{+}$, and $\sigma_{-}$are defined by $\sigma_{i j}=P_{l a b}^{2} \rho_{i j} \frac{\mathrm{d} f}{\mathrm{dm}}$. The data shown are for $4 \mathrm{GeV} / \mathrm{c}$ and $0.08 s^{-t} \leq 0.20 \mathrm{GeV}^{2}$. This same experiment acruired precise data on $\rho^{\circ}$ production by pions and $K^{* 0}, \bar{K}^{* 0}$ production by kaons, and permitted a systematic study of vector-meson production within the framework of SU-(3) symmetric, strongly absorbed Regge-pole models. New constraints to such amplitude analyses were provided by the $p-w$ interference data and by $w$-production data from the $Z G S$ Charged-Neutral Spectrometer. (From E-2ó8: see ref. 3.) 


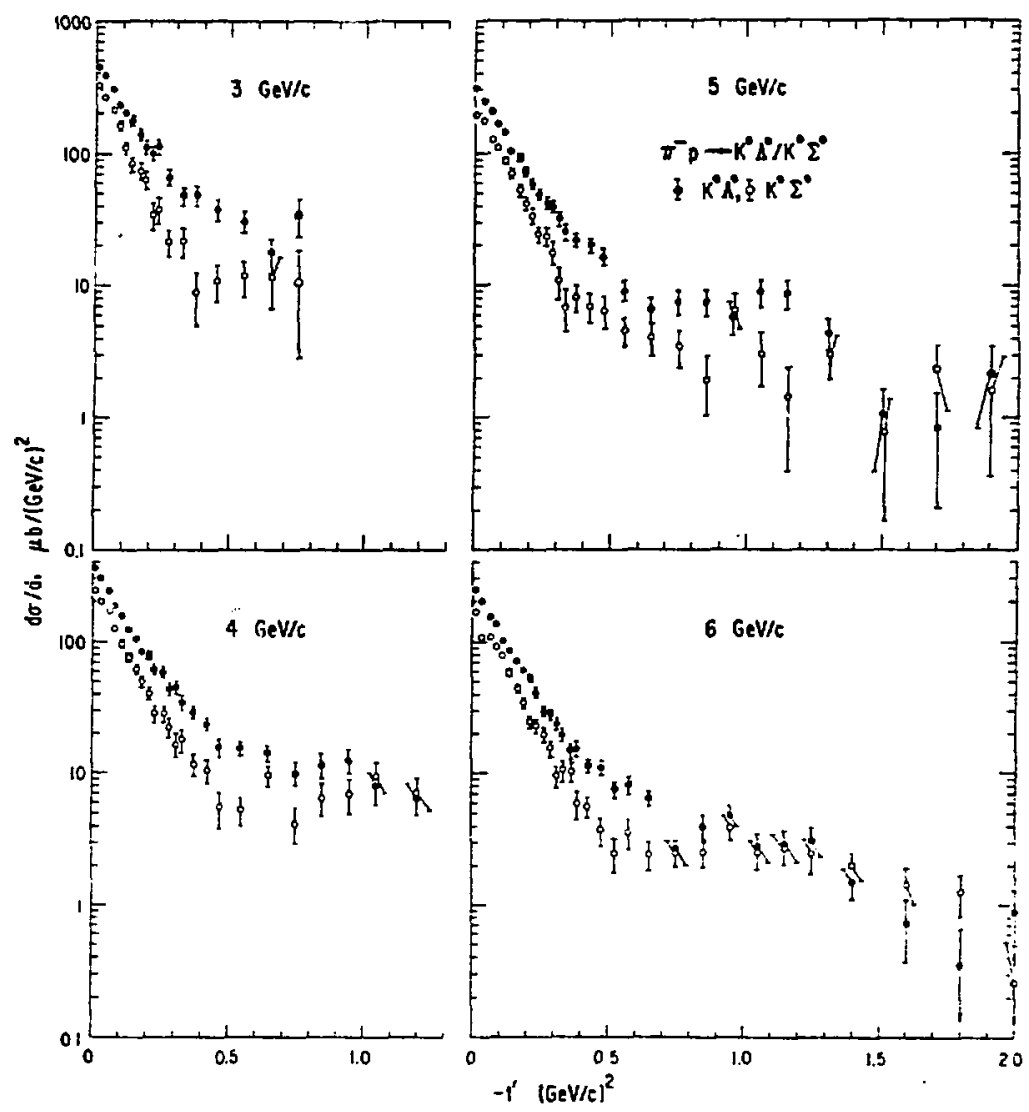

Fig. 7: Differential cross sections for $\pi^{-} \mathrm{p} \rightarrow \mathrm{K}^{0} \Lambda$ and $\pi^{-} \mathrm{p} \rightarrow \mathrm{K}^{0} \Sigma^{0}$ from 3 to $6 \mathrm{GeV} / c$. (From E-2Tl: see ref. 4.) 


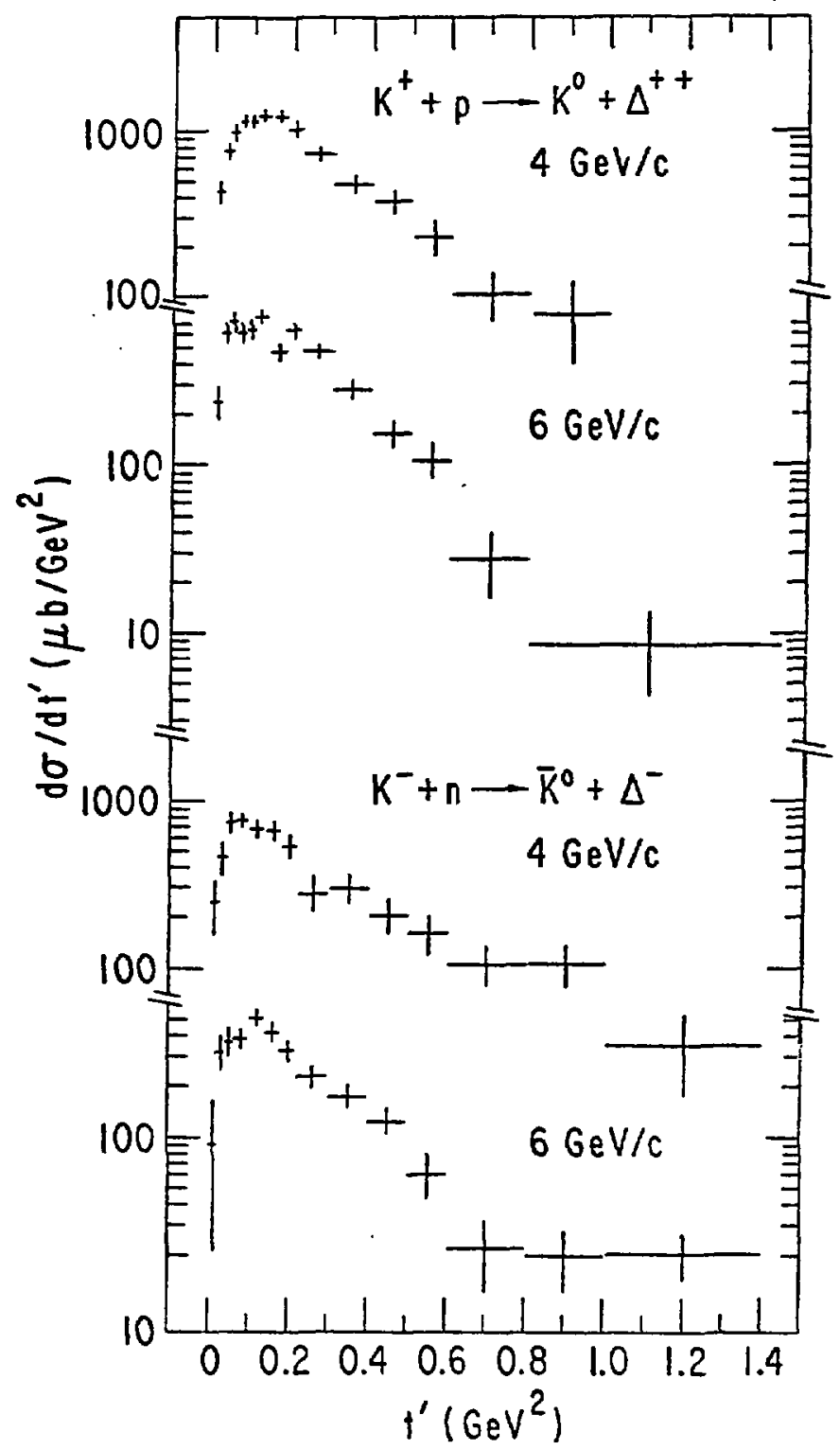

Fig. 8: Differential cross sections for line-reversal related reactions. The ratios of the $K^{0} \Delta^{++}$to $\bar{K}^{0} \Delta^{-}$cross sections at 4 and $6 \mathrm{GeV} / \mathrm{c}$ are $1.62 \pm 0.27$ and $1.60 \pm 0.26$ respectively. (From E-33l: see ref. 5.) 


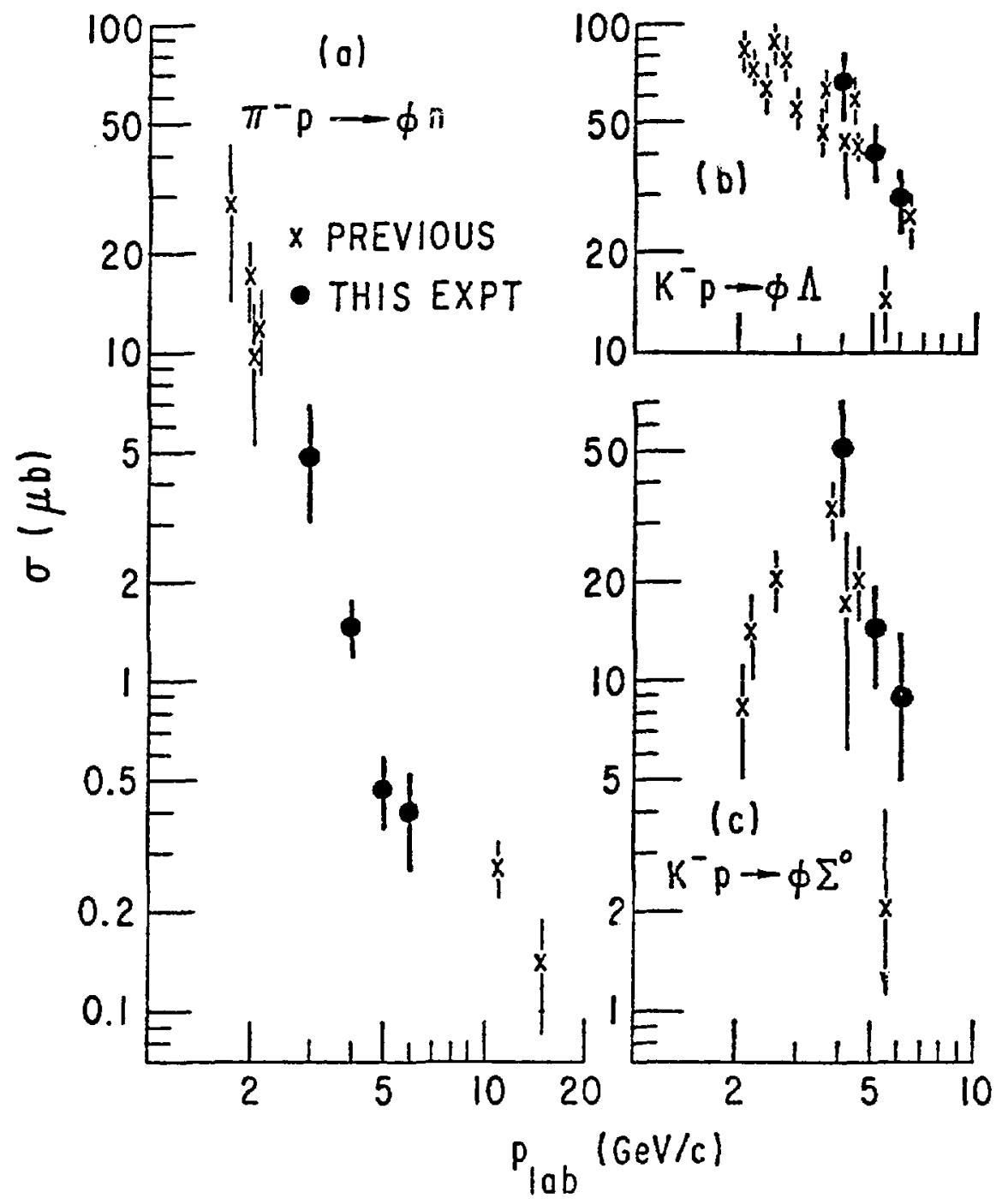

Fig. 9: Differential cross sections for $\phi$-meson production in $\pi$ " $p$ and $\mathrm{K}^{-} \mathrm{P}$ interactions. In (a), cross sections have been integrated out to $-t^{\prime}=0.9 \mathrm{GeV}^{2}$; in (b) and (c), cross sections are integrated over the entire angular range. (From E-325: see ref. 6.) 


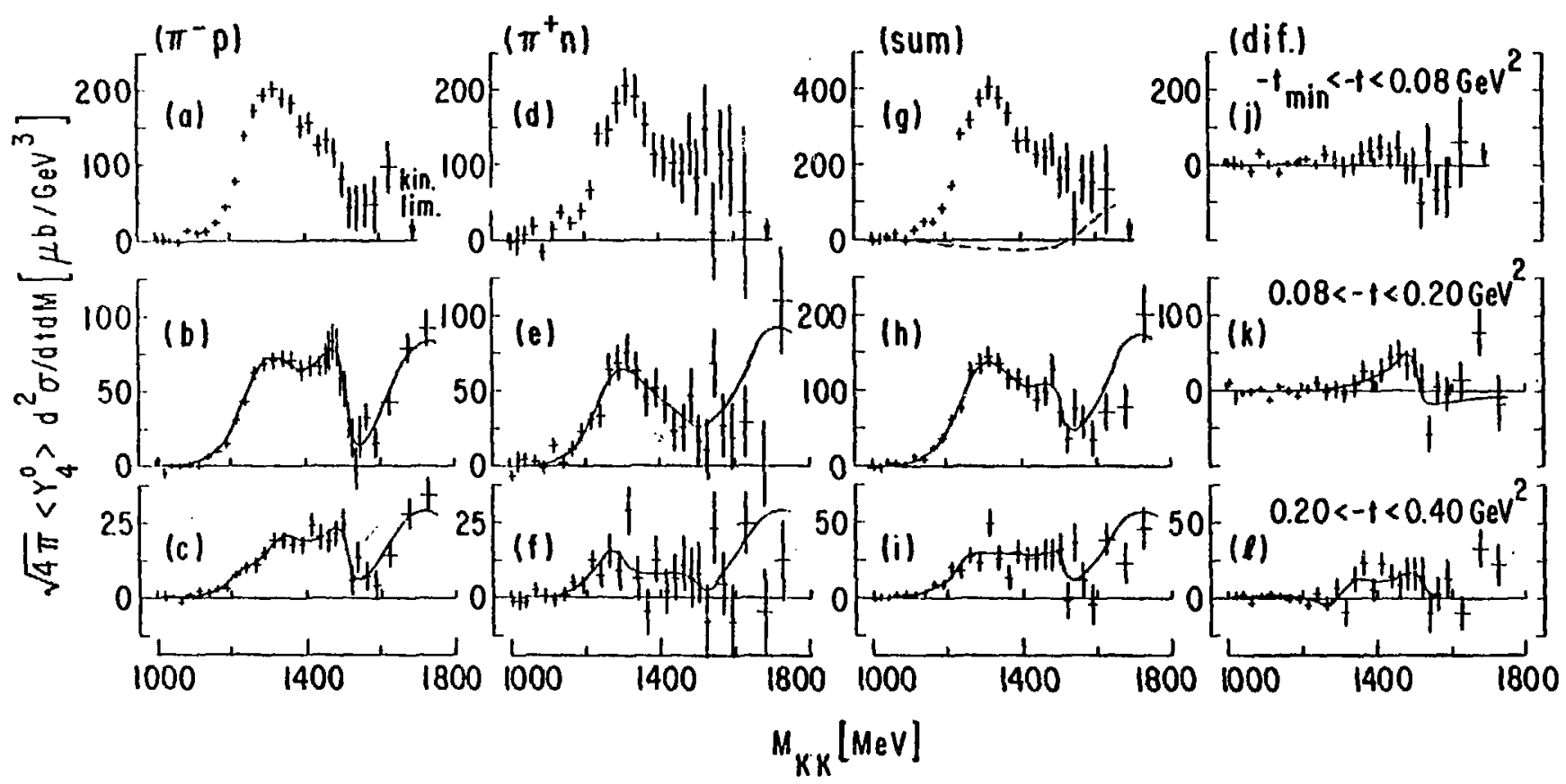

Fig. 10: The $Y_{4}^{0}$ moments of the $\mathrm{K}^{+} \mathrm{K}^{-}$decay angular distribution versus $\mathrm{K}^{+} \mathrm{K}^{-}$effective mass, for the reactions $\pi^{-} p+K^{-} K^{+} n$ and $\pi^{+} n \rightarrow K^{-} K^{+} p$ at $6 \mathrm{GeV} / c$, for the three $t$ ranges given. Parts (g) through (1) show the sums and differences of the moments for the two reactions, and isolate the interference of isospin-0 and isospin-1 $\mathrm{K}^{+} \mathrm{K}^{-}$otates. Production of $\mathrm{S}^{*}, \phi, f$, and $\mathrm{A}_{2}$ mesons and their mutual interferences were clearly observed in this experiment, and $f^{\prime}$ production by pions was detected for the first time through the interference of the $f$ and the $\mathrm{f}^{\prime}$, as seen in the dramatic dropoff around $1500 \mathrm{MeV}$. (From E-358: see ref. 7.) 

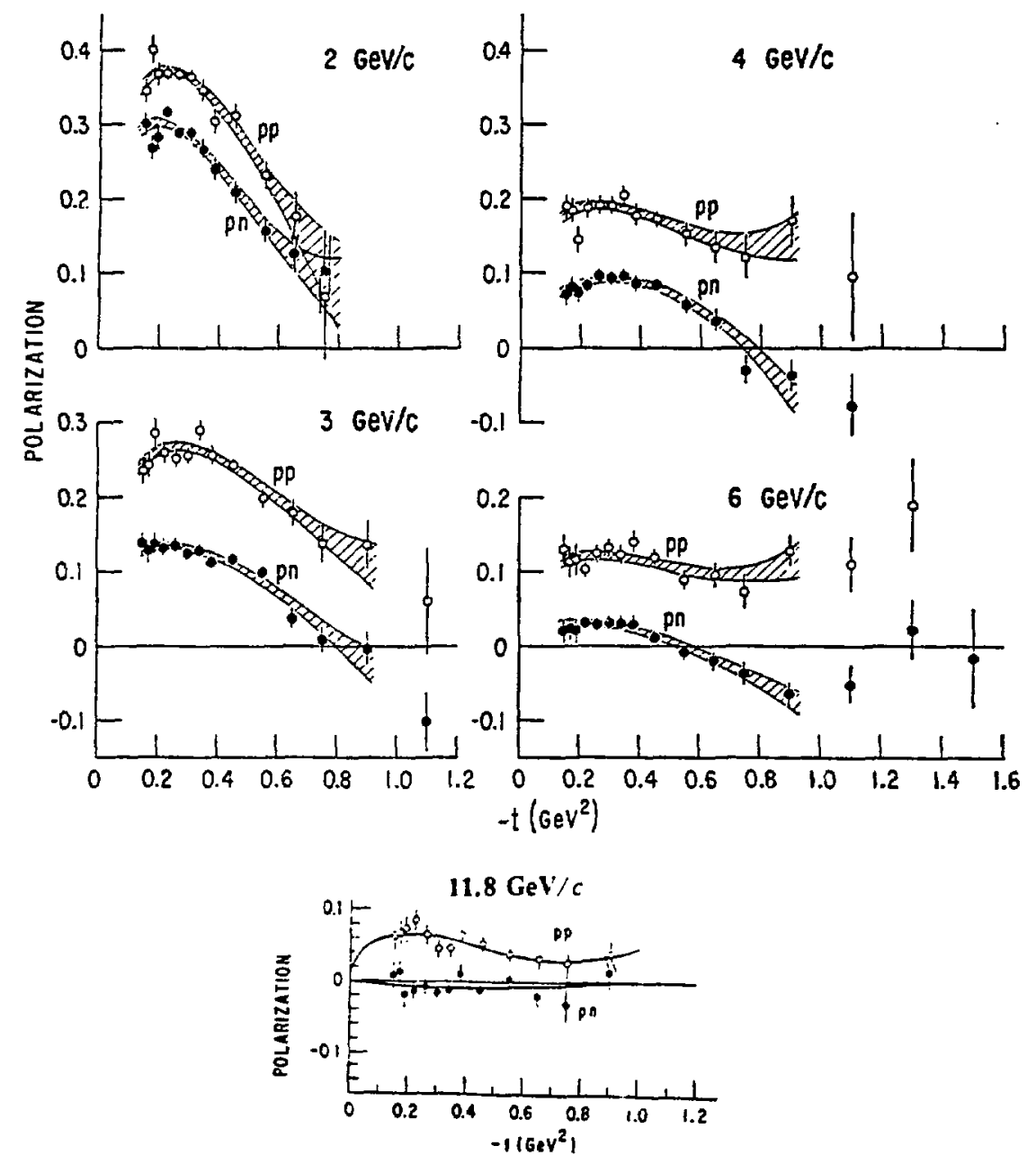

Fig. 11: Polarization asymmetries for pp and pn elastic scattering from 2 to $11.8 \mathrm{GeV} / \mathrm{c}$. Fits to the data from 0.15 to $1.0 \mathrm{GeV}^{2}$ are shown as bands ( \pm 1 standard deviation). These data allowed for the first time the separation of isospin- 0 and isospin-l $t$-channel exchange contributions to the single-flip amplitude, and revealed a previously unsuspected rapid energy dependence in the isospin-0 amplitude. (From E-376 and E-391: see ref. 8, 9.) 


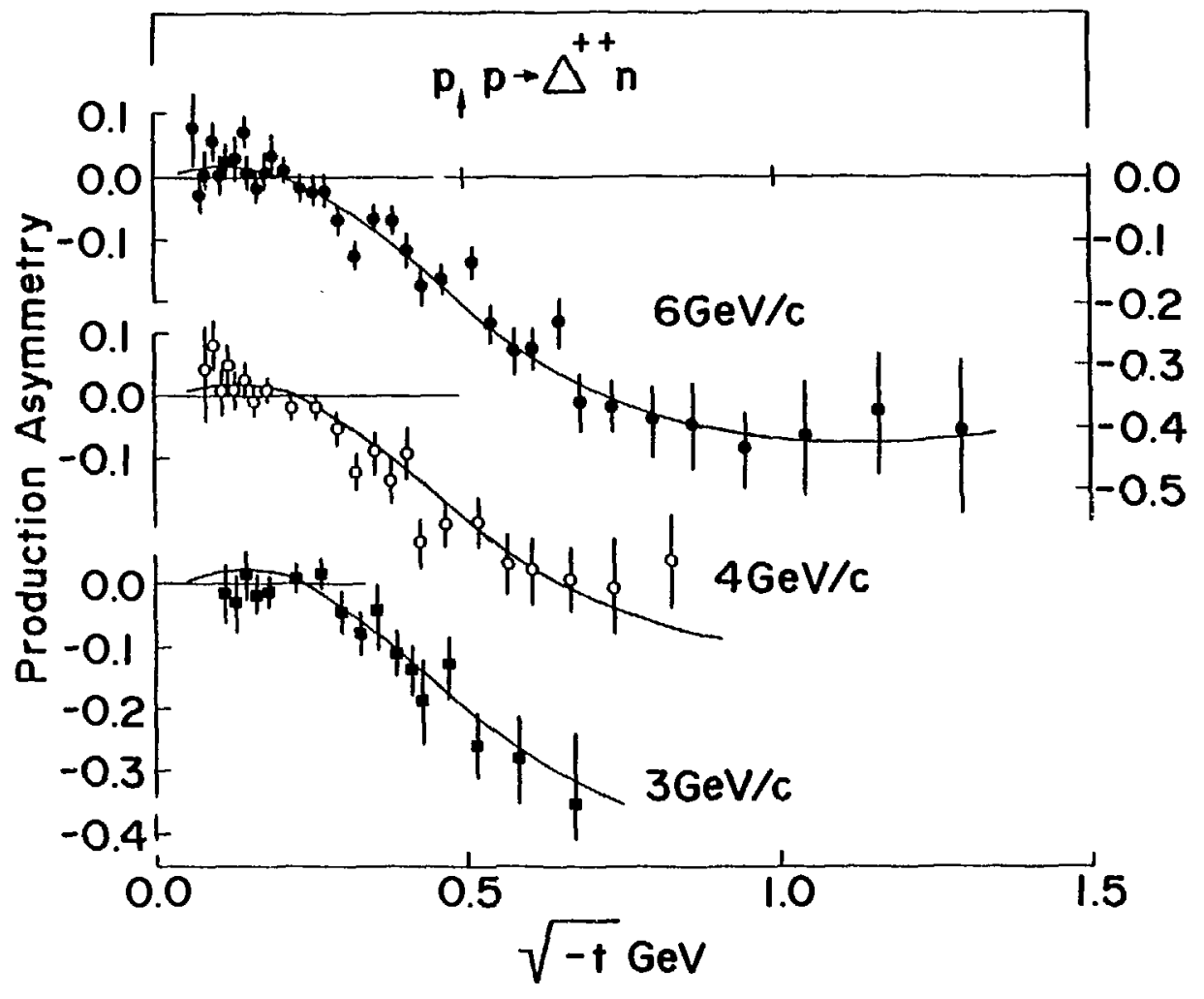

Fig. 12: Overall left-right asymmetries in $p, p \rightarrow \Delta^{t+} n$ at 3,4 , and $6 \mathrm{GeV} / \mathrm{c}$. The curve which is ref. 10 .) With all three data sets is an eyeball interpolation of the $6 \mathrm{GeV} / \mathrm{c}$ data. (From E-339: see 